\title{
DAM and automated digital video workflows
}

\section{Mukul Krishna}

is responsible for the digital media practice at Frost \& Sullivan. He has written extensively on the enterprise content management, digital asset management, video server, video streaming and encoding services, and interactive kiosk markets. He has also led consulting projects in these fields for key clients, has spoken at various speaking engagements on his area of expertise, and is widely quoted in the press, including major publications such as Forbes and the Washington Post.

\section{David Heppe}

is responsible for running the Workgroup and Enterprise Solutions business at Telestream, a media encoding and workflow automation provider. Following a 7-year history guiding the company's worldwide sales and marketing programs, Mr. Heppe recently stepped up to President. He has spent nearly 20 years establishing and running business units and sales organizations for companies specializing in computer, telecommunications and video technology. Mr. Heppe holds engineering degrees from the University of Nevada and Stanford, as well as an MBA from Harvard Business School.

Keywords: Integrated asset management, transcoding, workflow automation, centralization, graphics workflow enabler, digital distribution

Abstract With the digitization of the broadcast space and the growing number of digital distribution outlets and viewing options available to today's consumers, the need to effectively manage video assets has never been higher. Digital asset management has become a must-have solution for entities wanting to create greater efficiencies in production, post-production, management, and delivery that ultimately improve the bottom-line. When integrated within an automated file-based video workflow, asset management provides incredible cost savings and efficiencies. Integrating workflow automation into content delivery and facility ingest is analyzed. New graphics workflow enablers that provide the ability to cost-effectively generate customized video content for the growing number of distribution outlets are also examined. Journal of Digital Asset Management (2006) 2, 163-167. doi:10.1057/palgrave.dam.3650024

David Heppe

President (Telestream, Inc.) Tel: +15304701323 Fax: +15304701300

E-mail:

davidh@telestream.net
Digital asset management (DAM) has come a long way since its inception as a market in the 1990s. Gone are the days when it was perceived as a "nice-to-have" solution, as the digital revolution has pervaded almost every aspect of our lives. Thanks to the digitization of the broadcast space and the advent of technology to stream video from the web to handheld devices, the consumer just can not get enough.

The need to manage this ever-growing demand for video has now made asset management a "must have" solution for entities looking at effectively managing their fastgrowing repositories of video to create greater efficiencies in production, post-production, repurposing, management, and delivery, and the monetization that ultimately adds to their bottom-line in an extremely competitive and cut-throat marketplace.
DAM systems help the digitized workflow all the way from encoding and transcoding in the right format to ingest; search and retrieval; browsing, annotation; repurposing and delivery.

Branded in all flavors such as rich asset management, media asset management, brand resource management, digital media management, enterprise content management among others, DAM has now started being seen as an incredible cost saving center when integrated within a digital video workflow with encoding/transcoding engines and nonlinear editors.

Studies conducted by Frost and Sullivan have shown how deploying these systems is no longer a "leap-of-faith" as it once was. Now there are very tangible numbers available in the market that justify the attractiveness of a business case for deployment and even more so when 
dealing with time-based dynamic content such as video.

\section{WHAT IS THE VALUE PROPOSITION?}

Increasingly users have understood the value offered through the digitization of their media assets. The value is uniquely, but not mutually exclusively, tied-in to all aspects of the value chain, namely: logging/ingest, asset management, delivery, rights management and online content management.

At one end of the value chain ingest engines add value to the digital asset by creating a process to encode and transcode in the correct format and then to index and provide metadata to a DAM system for searching and retrieving the asset. This is a great time saver when it comes to production time and in turn reduces overhead costs significantly. The process also enables repurposing of digital assets that is of great value to content owners from corporate entities to studios that have huge repositories of their branded electronic assets that need to be catalogued. Just the cost savings in terms of real estate and maintenance can be very significant in moving to a digital archive from a physical archive.

Beyond logging and at the core is the DAM process. Increasingly enterprises have moved towards centralization of their assets as they find it easier to manage digital assets and track them from a single location. This has created a great demand for efficient processes to manage these assets. The cost of lost or misplaced work has become a major cause of concern for corporations as the cost effectiveness associated with reusing and repurposing existing assets, as well as maintaining a homogenous message, has become clear.

Delivery of digital assets is fast becoming a part of the core asset management solution as well. With increased regional centralization of large functional workgroups in media production, the need for a high degree of collaboration to get the finished product has become paramount. Although broadcasters and advertisers realize efficiencies by centralizing the preparation of content to one facility, other production work, such as graphic art, may be done at another facility. As companies add more and more rich digital media to their brand strategies, file sizes are exploding. And for finished assets, as delivery methods and viewing choices continue to evolve and expand for the consumer, multiformat encoding solutions are required for ultimate distribution.

Digital media files, particularly video, have become so large that simple FTP services across the public Internet are not acceptable. By employing digital delivery services, dispersed workers can easily work and access the same assets from different locations and in so doing, also cut down the time-to-market significantly.

\section{WHAT ABOUT THE RETURN ON INVESTMENT?}

On the most micro-level Frost and Sullivan has seen the tremendous appeal that these integrated systems have on even the most mundane tasks. For example, an experienced and fully burdened editor in Burbank, California who costs around $\$ 200$ an hour and doing tasks that could easily be automated, or at the very least be digitized, can provide effective time savings that translate into cost savings as well as the potential to generate revenue.

If we look at the same editor and add an integrated asset management system that helps in chopping off one hour a day spent in search and retrieval, it saves the company $\$ 52,000$ a year, assuming a work year consists of 260 days. That also gives the editor $260 \mathrm{~h}$ that he/she can use towards more productive tasks.

Add to this equation the time this editor might also be spending doing other mundane tasks that could be automated, such as:

- adding logos, fade-in/fade-out every $5 \mathrm{~s}$, adding animation and promos in the cable space

- adding seasonal logos and promotions in the Video on Demand space

- transcoding and burn-in sub-titling in the broadcast and mobile space

- security coding added to video before transmission in the entertainment space

Suddenly, the $\$ 52,000$ saving figure starts climbing up into the hundreds of thousands as these tasks, personnel assigned to these tasks, the time taken to complete them and the amount of video scales up.

The unfortunate reality is that many companies still do it the old way and rob 
themselves of higher profits and growth as these costs cut into their ability to be competitive. All this can be avoided by a simple investment into the integration of automation into the digital workflow.

\section{WHO DOES THAT?}

There are now an increasing number of workflow automation enablers available in the market that help provide these sorts of capabilities. Companies like Telestream have evolved their media transcoding engines into fully integrated workflow automation solutions that address a growing number of needs, from DAM archives to broadcast to graphics and beyond.

Before looking at the specifics of automating graphics tasks, let's look at another workflow, which has revolutionized the distribution end of the media and entertainment (M\&E) space.

\section{AUTOMATING COMMERCIAL WORKFLOWS}

The creation, management, distribution, and play out of commercials are the lifeblood of the M\&E world - after all, commercials pay the bills. On the production side DAM solutions are becoming sophisticated enough that, when combined with efficient ingest and transcoding engines, they can effectively organize and provide easy on-line access to commercials for ad agencies, clients, and the post-houses themselves. Indeed, review-and-approval solutions enabled by DAM systems are continually becoming more practical and efficient business tools.

Until very recently, managing the flow of the commercials at the broadcast, cable or other distribution facility remained cumbersome and manual. Traditionally, commercials were delivered to TV stations via tape. Large tape duplication facilities generated thousands of tapes every day and shipped them to the stations. Some years ago, digital delivery services arose, offering distribution of commercials as files (typically via IP satellite). These services installed dishes and catch servers at broadcast facilities. The delivery services quickly grabbed a large market share from the tape distributors, offering greatly reduced distribution costs to the ad agencies, enabled by the replacement of tapes and couriers.
So, the delivery services were winners, and the agencies were winners, but what about the broadcast and cable companies? Their workflows changed very little. They received a digital file, which was often incompatible with other digital systems in the facility. So the file was dubbed to tape which could be loaded into the playout server or routed as a video signal through the facility to the server. Since commercials were coming from different sources, each one had to be taken through a visual and audio quality assurance process to ensure appropriate signal levels. Operators needed to learn how to operate each delivery service's catch server and collect unique metadata from each. Finally, that metadata had to be matched with in-house metadata (House ID) and dialed into the automation system controlling the actual playout of the commercial at the appropriate time.

This very complex workflow required a great deal of manual processing, subject to high costs and human error.

Digital workflow automation enablers have provided tremendous value to this ingest/playout workflow. Catch servers are monitored for the arrival of new content. Associated metadata files are similarly ingested. Built-in transcoding enables first-pass audio level analysis for automated quality assurance, and creation of proxies and thumbnails for desktop review. All incoming commercials are consolidated and accessible through a single, searchable user interface. Traffic systems can be queried for House ID. Transcoding can be invoked automatically or manually to deliver the media in the required format to the playout server. Finally, the automation system can be notified to play out the commercial when that particular commercial's House ID has been scheduled. What had been a manual and cumbersome process is now automated, reducing costs and increasing reliability (Figure 1).

Current generation workflow automation systems were developed by brute force vendors exchanging proprietary API and metadata information with each other, writing plug-ins and integration code, testing in the lab, testing in the field, dealing with the field consequences of software revisions occurring without warning, and the list goes on and on. Next generation services will benefit greatly from the growing implementations of web 


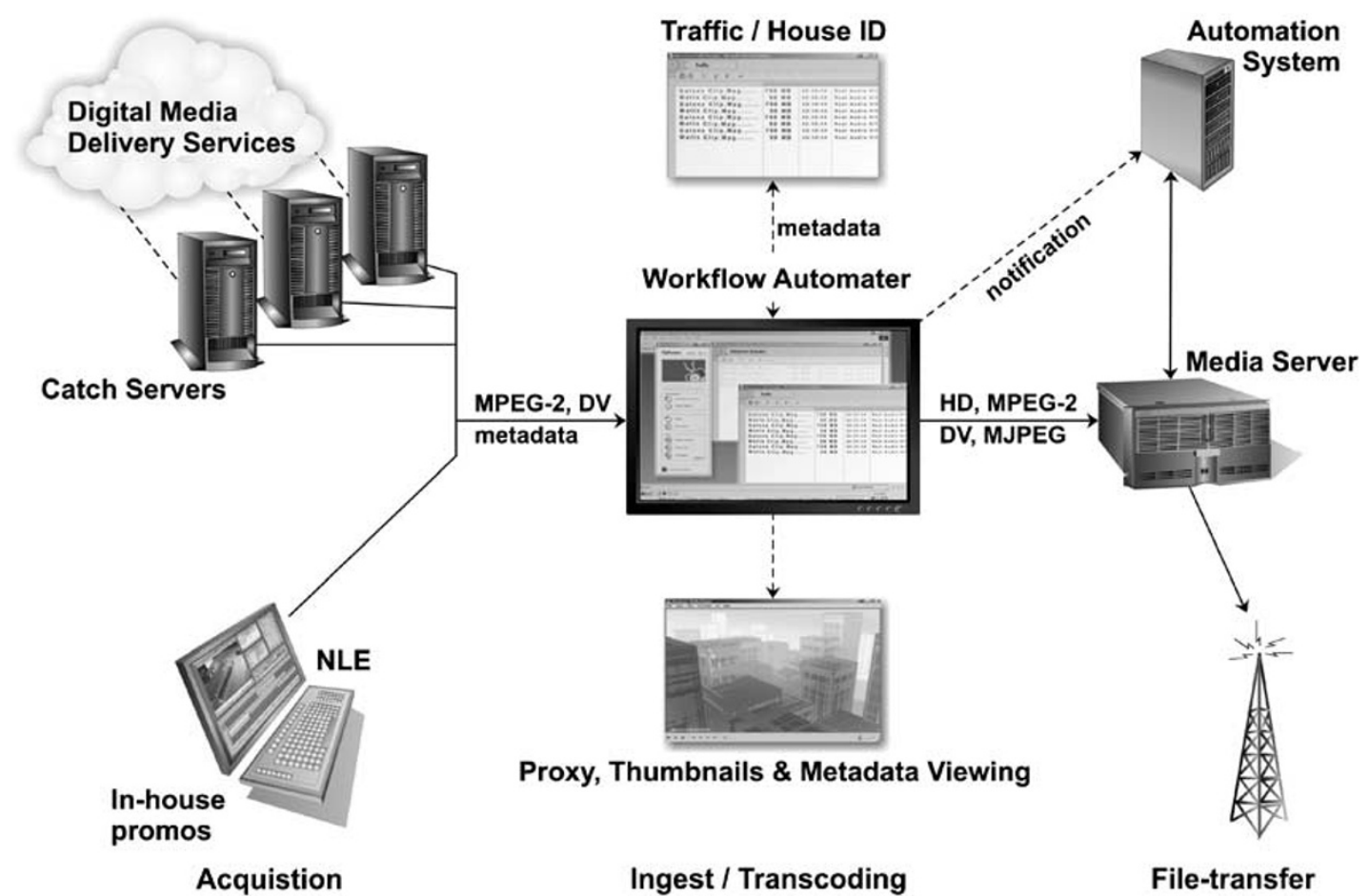

Figure 1: Commercial workflow automation in a typical broadcast or cable facility

services, open APIs, and more common file format wrappers such as MXF. In turn, the end users benefit from lower vendor costs to implement and maintain these workflow enabler systems.

\section{BRANDING AND THE EXPANDING DISTRIBUTION LANDSCAPE}

Content owners and distributors are now challenged by the rapid expansion of media distribution outlets. The number of ways that consumers can access and view content has grown to include traditional broadcast (HD \& $\mathrm{SD})$, cable VOD, IPTV, web, mobile, podcasting, kiosks, gas pumps, electronic billboards, and a growing number of personal media players. Some content owners try to handle as much as they can in-house. Others turn to content aggregators to adapt to the ever-changing landscape, including rights management, format transcoding, billing, security, etc. In either case, the extent to which the repurposing of content into multiple formats and brands can be automated, as well as the addition of transitions and promos, the whole workflow becomes more affordable and reliable. Effectively handling these workflow challenges can result in significant new revenue opportunities with affordable cost structures.
In our original example, we discussed the high costs involved in using a highly skilled editor to execute mundane graphics tasks like the addition of logos and repetitive fade-in and fade-outs. By utilizing a file-based, automated workflow enabler, the editor can create an "effects" factory or template with predefined graphics configurations. Then, a data entry operator can simply add the metadata, such as "when" and "where" to apply the effects. In some cases metadata can be pulled from databases (e.g. "Coming next ...”). enabling completely automated processing. Simple fade-ins/fade-outs of graphics have been relatively easy, but with solid transcoding and effects engines at the core, video files can now be combined - squeezing one to a corner while fading in a second. Whole blocks of programming and promotions can be created and set up for play out, as well as being transcoded and re-branded for multiple distribution outlets. Using file-based, rather than baseband video processes, makes it easy for an operator to execute these tasks (Figure 2).

The new graphics workflow enablers provide the ability to generate customized video content in a far less expensive way. In a decade that is 


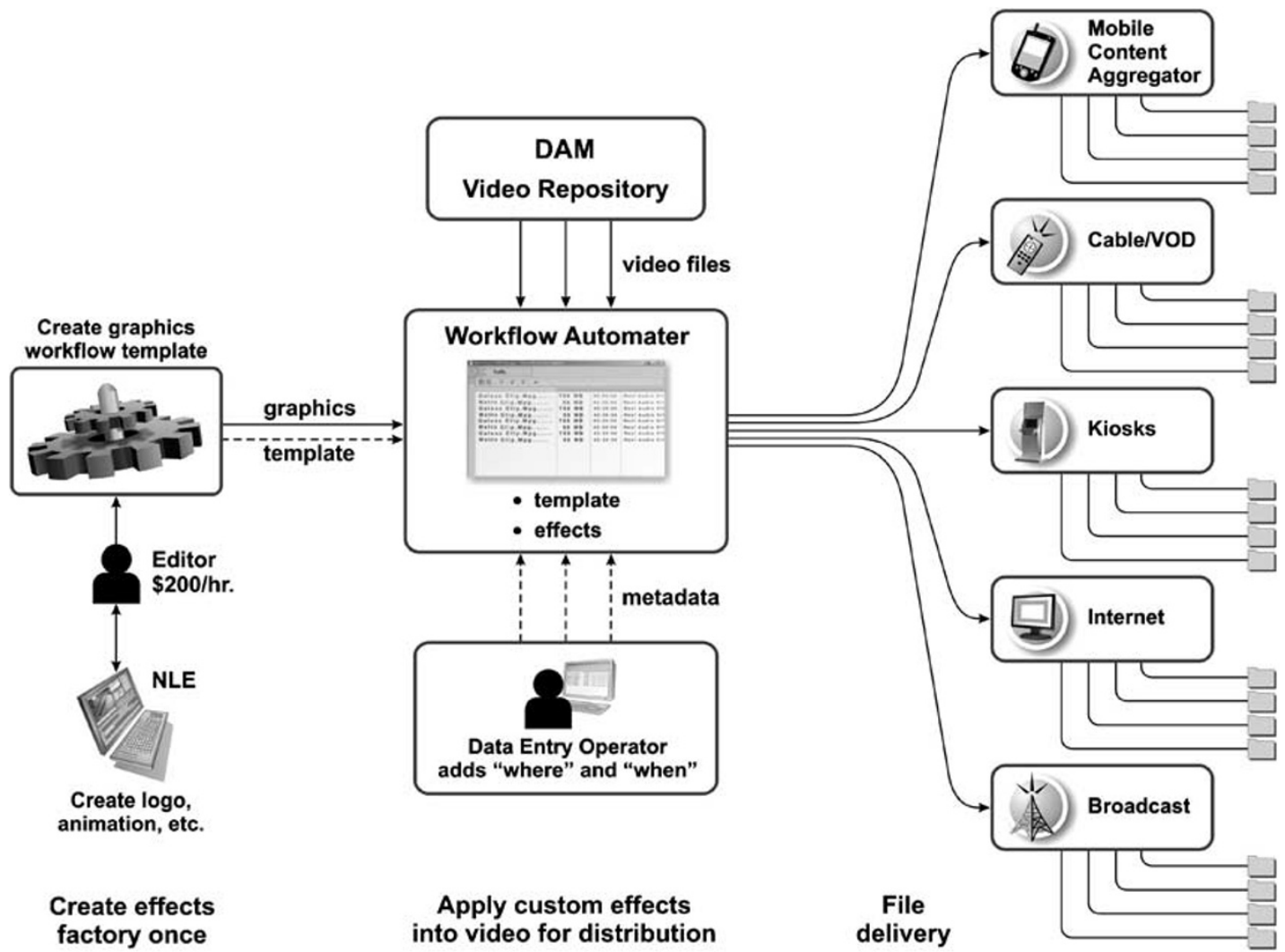

Figure 2: Sample graphics workflow automation

being defined by outsourcing, these tasks can now be transferred from highly skilled editors to data entry operators. In doing so a more consistent output emerges, and the editor's time is freed up for more creative tasks. The integration of workflow automation opens up new opportunities for quick video customization and distribution via new avenues at affordable prices.

\section{WHERE IS IT ALL HEADING?}

With the growing number of digital media distribution methods and players for viewing content, the number of distribution formats is not decreasing. Today's transcoding engines are evolving into workflow automation engines that handle multiformat production capability along with branding, DRM, delivery, etc.

As home computing power increases, many of the workflow engine's reformatting tasks will take place behind the scenes in the living room - enabling consumers to control how and where they view broadcast and VOD content along with their own personal content. Choices will include the places and devices they want to use for viewing their digital media. At this point, automated digital workflows migrate from central DAM systems to personal asset management (PAM) systems in the home.

This article has only scratched the surface of how a truly automated digital video workflow will not only significantly drive down costs but also help in added revenue generation. Even so, through the examples we have seen, it is apparent how a well-integrated DAM system can help users save resources. Every few months the market is made aware of newer and more creative ways in which enterprising users have made DAM work for them in ways even the vendor community had not imagined.

Vendors such as Telestream are helping change the landscape and paving the way for more efficient and user-friendly next generation video workflows. As we start moving into a truly digital age, characterized by convergence and outsourcing, such systems will go a long way in helping the user community realize the true potential of the technology innovation that has made automating digital video workflows possible. 